\title{
COUNTABLE SECTIONS FOR LOCALLY COMPACT GROUP ACTIONS. II
}

\author{
ALEXANDER S. KECHRIS
}

(Communicated by Andreas R. Blass)

\begin{abstract}
In this paper we study the structure of the orbit equivalence relation induced by a Borel action of a second countable locally compact group on a standard Borel space.
\end{abstract}

Let $G$ be a second countable locally compact group and $(g, x) \mapsto g . x$ a Borel action of $G$ on a standard Borel space $X$. (A standard Borel space is a Polish space with the associated Borel structure, and an action is Borel if the function $(g, x) \mapsto g . x$ is Borel from $G \times X$ into $X$.) We denote by $E_{G}$ the equivalence relation induced by this action, i.e., $x E_{G} y \Leftrightarrow \exists g \in G(g . x=y)$. It is well known that $E_{G}$ is a Borel equivalence relation on $X$.

A Borel equivalence relation $E$ on a standard Borel space $X$ is called countable if every equivalence class $[x]_{E}$ of $E$ is countable. Given two Borel equivalence relations $E, E^{\prime}$ on $X, X^{\prime}$ resp., we say that $E$ is (Borel) reducible to $E^{\prime}$, in symbols $E \leq E^{\prime}$, if there is a Borel map $f: X \rightarrow X^{\prime}$ such that $x E y \Leftrightarrow f(x) E^{\prime} f(y)$, and we say that $E, E^{\prime}$ are bireducible, in symbols $E \approx^{*} E^{\prime}$, if $E \leq E^{\prime}$ and $E^{\prime} \leq E$. It has been shown in [DJK] that bireducibility for countable Borel equivalence relations is also equivalent to the following notion: We say that $E, E^{\prime}$ are stably isomorphic, in symbols $E \cong{ }^{s} E^{\prime}$, if there are Borel sets $A \subseteq X, B \subseteq X^{\prime}$ which meet every $E$ - (resp. $E^{\prime}$-) equivalence class (such sets are called complete sections or full) and $E\left|A \cong E^{\prime}\right| B$, where $E \mid A$ denotes the restriction of $E$ to $A$ and $\cong$ is (Borel) isomorphism. (Two Borel equivalence relations $F, F^{\prime}$ on standard Borel spaces $Y, Y^{\prime}$ resp. are (Borel) isomorphic if there is a Borel bijection $f: Y \rightarrow Y^{\prime}$ with $x F y \Leftrightarrow f(x) F^{\prime} f(y)$.) Denoting by $I_{S}$ the transitive equivalence relation $S \times S$ on $S$, it turns out that we have, for countable $E, E^{\prime}$,

$$
E \approx E^{\prime} \Leftrightarrow E \cong{ }^{s} E^{\prime} \Leftrightarrow E \times I_{\mathbb{N}} \cong E^{\prime} \times I_{\mathbb{N}}
$$

where as usual the product of two equivalence relations $E, F$ is the equivalence relation

$$
(x, y)(E \times F)\left(x^{\prime}, y^{\prime}\right) \Leftrightarrow x E x^{\prime} \& y F y^{\prime} .
$$

We now state our main results in this paper.

Received by the editors April 14, 1992.

1991 Mathematics Subject Classification. Primary 03E15.

Research partially supported by NSF grant DMS-9020153. (C) 1993 American Mathematical Society
$0002-9939 / 93$ \$1.00 + \$.25 per page 
Theorem 1. Let $G$ be a second countable locally compact group acting in a Borel way on a standard Borel space $X$. Then there is a unique decomposition $X=$ $C \cup U$ into invariant Borel sets such that $E_{G} \mid C$ is countable and $E_{G} \mid U \cong F \times I_{\mathbb{R}}$, with $F=E_{G} \mid Z$ for $Z$ a complete Borel section of $E_{G} \mid U$ such that $E_{G} \mid Z$ is countable.

We call $E \mid C$ the countable part of $E_{G}$ and $E_{G} \mid U$ the uncountable (or continuous) part of $E_{G}$. For the latter we have the following additional information.

Theorem 2. The map $F \mapsto F \times I_{\mathbb{R}}$ gives a one-to-one correspondence between: (1) countable Borel equivalence relations up to stable isomorphism, and (2) Borel equivalence relations of the form $E_{G}, G$ second countable locally compact, with all equivalence classes uncountable, up to isomorphism.

These results provide pure Borel theoretic versions of analogous results of Feldman-Hahn-Moore [FHM, §§4-6], who study nonsingular measurable actions of second countable locally compact groups on standard measure spaces. The proofs of Theorems 1 and 2 use, among other things, a result in [K], which provides a purely Borel theoretic extension of the main result of [FHM], concerning the existence of countable complete Borel sections in Borel actions of such groups.

In the case of $\mathbb{R}$-actions, i.e., flows, one can obtain further information using [W] (see also [K]) and one of the results in [DJK]. Note that if $E_{\mathbb{R}}$ is the orbit equivalence relation of a flow, then every equivalence class is either a singleton or uncountable (this is because the stabilizer of a point under the action is a closed subgroup of $\mathbb{R}$; see [V]). Recall that a Borel equivalence relation $E$ on a standard Borel space $X$ is called smooth if there is a standard Borel space $Y$ and a Borel map $f: X \rightarrow Y$ with $x E y \Leftrightarrow f(x)=f(y)$. For $E$ of the form $E_{G}$, this is equivalent (as it follows from [B], see also [K]) to saying that there is a Borel set $A \subseteq X$ picking exactly one element out of each $E$-equivalence class. It is easy to classify $E_{\mathbb{R}}$ up to Borel isomorphism when it is smooth. For nonsmooth ones we have

Theorem 3. Let $E_{\mathbb{R}}^{1}, E_{\mathbb{R}}^{2}$ be two nonsmooth Borel equivalence relations, induced by Borel actions of $\mathbb{R}$. Let $c_{i}=$ the cardinality of the set of singleton equivalence classes for $E_{\mathbb{R}}^{i}$. Then $E_{\mathbb{R}}^{1} \cong E_{\mathbb{R}}^{2} \Leftrightarrow c_{1}=c_{2}$. In particular, any two nonsmooth equivalence relations induced by Borel flows with no fixed points are Borel isomorphic.

It should also be true that any two nonsmooth $E_{G}$, with $G$ amenable, which have all equivalence classes uncountable are Borel isomorphic, but this is an open problem.

Added in proof. This has now been verified by Jackson, Kechris, and Louveau for compactly generated $G$ of polynomial growth, extending a result of B. Weiss.

\section{Some Lemmas}

The following lemmas are needed for the proofs of the main results.

Lemma 1.1. Let $Z, X$ be standard Borel spaces, $H \subseteq Z \times X$ a Borel set with $H_{z}=\{x:(z, x) \in H\} \neq \varnothing$, for every $z \in Z$. Assume to each $z \in Z$ we have assigned $a$-ideal $I_{z}$ of subsets of $H_{z}$ (i.e., a collection containing $\varnothing$ and 
closed under subsets and countable unions) such that:

(1) $H_{z} \notin I_{z},\{x\} \in I_{z}$ for every $x \in H_{z}$;

(2) For each Borel $P \subseteq H$, the set $P_{I}=\left\{z \in Z: P_{z} \in I_{z}\right\}$ is Borel.

Then there is a Borel map $\varphi: Z \times \mathbb{R} \rightarrow X$ such that for each $z \in Z, \varphi_{z}: \mathbb{R} \rightarrow$ $X$ given by $\varphi_{z}(r)=\varphi(z, r)$ is a Borel isomorphism of $\mathbb{R}$ with $H_{z}$.

Proof. Since $H$ is Borel, we can find a family $\left\{H^{s}\right\}$, where $s$ varies over the set $\mathbb{N}^{<\mathbb{N}}$ of finite sequences from $\mathbb{N}$, such that:

(i) $H^{s}$ is Borel;

(ii) $H^{\varnothing}=H, H^{s^{\wedge} n} \cap H^{s^{\hat{m}}}=\varnothing$, if $n \neq m, H^{s}=\bigcup_{n} H^{s^{\wedge} n}$;

(iii) If $\alpha \in \mathbb{N}^{\mathbb{N}}$ and $H^{\alpha \mid n} \neq \varnothing$ for all $n$, then $H^{\alpha}:=\bigcap_{n} H^{\alpha \mid n}$ is a singleton $\{w\}$ and if $w_{n} \in H^{\alpha \mid n}$, ihen $w_{n} \rightarrow w$.

(We view here $Z, X$ and thus $Z \times X$ as Polish spaces, so $w_{n} \rightarrow w$ means convergence in $Z \times X$.)

Put now $H_{z}^{s}=\left\{x:(z, x) \in H^{s}\right\}$. Then it is easy to see that $\left\{H_{z}^{s}\right\}$ has properties (i)-(iii) for $H_{z}$.

For each $z$ now define a tree $T_{z}$ on $\mathbb{N}$ (i.e., a set of finite sequences from $\mathbb{N}$ closed under initial segments) as foliows: $s \in T_{z} \Leftrightarrow H_{z}^{s} \notin I_{z}$. Since every singleton is in $I_{z}$, it follows that

$$
\forall s \in T_{z} \exists s^{\prime}, s^{\prime \prime} \in T_{z}\left[s \subseteq s^{\prime}, s \subseteq s^{\prime \prime} \text {, and } s^{\prime}, s^{\prime \prime} \text { are incompatible }\right] .
$$

(Otherwise for some $s \in T_{z}$ there is $\alpha \in \mathbb{N}^{\mathbb{N}}, s \subseteq \alpha$, such that $H_{z}^{\alpha \mid n} \notin I_{z}$ for all $n$, but if $t \supseteq s, t \in \mathbb{N}^{\mathbb{N}}$, and $t \neq \alpha \mid n$, then $H_{z}^{t} \in I_{z}$. Then $H_{z}^{s}=H_{z}^{\alpha} \cup\left\{H_{z}^{t}\right.$ : $\left.s \subseteq t, t \in \mathbb{N}^{n}, t \neq \alpha \mid n\right\}$, where $H_{z}^{\alpha}=\bigcap_{n} H_{z}^{\alpha \mid n}$. Thus $H_{z}^{\alpha} \notin I_{z}$, which is impossible since $H_{z}^{\alpha}$ is a singleton.)

Define now inductively on the length of $u \in 2^{<\mathbb{N}}$ a sequence $s_{u} \in T_{z}$, so that $s_{\varnothing}=\varnothing$ and $s_{u^{\wedge} 0}, s_{u^{\wedge} 1}$ are the first two (in some enumeration of $\mathbb{N}^{<\mathbb{N}}$ ) incompatible extensions of $s_{u}$ in $T_{z}$. Finally define for each $\alpha \in 2^{\mathbb{N}}$

$$
\tilde{\varphi}_{z}(\alpha)=\text { the unique element of } \bigcap_{n} H_{z}^{s_{\alpha \mid n}} .
$$

In view of property (2) of the assignment $z \mapsto I_{z}$, it is easy to check that the map $\tilde{\varphi}(z, \alpha)=\tilde{\varphi}_{z}(\alpha)$ from $Z \times 2^{\mathbb{N}}$ into $X$ is Borel. Moreover, it is clear that $\tilde{\varphi}_{z}: 2^{\mathbb{N}} \rightarrow H_{z}$ is a Borel injection. Let also $\tilde{\psi}: H \rightarrow 2^{\mathbb{N}}$ be a Borel injection, so that in particular $\tilde{\psi}_{z}: H_{z} \rightarrow 2^{\mathbb{N}}$ given by $\tilde{\psi}_{z}(x)=\tilde{\psi}(z, x)$ is a Borel injection. By the usual Schroeder-Bernstein argument applied to $\tilde{\varphi}_{z}, \tilde{\psi}_{z}$ we can finally find a Borel map $\varphi: Z \times 2^{\mathbb{N}} \rightarrow X$ such that $\varphi_{z}$ is a bijection of $2^{\mathbb{N}}$ with $H_{z}$. Since $2^{\mathbb{N}}, \mathbb{R}$ are Borel isomorphic, this completes the proof.

Lemma 1.2. Let $E$ be a Borel equivalence relation on a standard Borel space $X$. Assume to each $E$ equivalence class $C$ we have assigned a $\sigma$-ideal $J_{C}$ of subsets of $C$ such that:

(1) $C \notin J_{C},\{x\} \in J_{C}$ for all $x \in C$;

(2) If $P \subseteq E$ is Borel, then the set $P_{J}=\left\{x: P_{x} \in J_{[x]_{E}}\right\}$ is Borel.

Let also $F$ be a countable Borel equivalence relation on a standard Borel space $Y$ and $f: X \rightarrow Y$ a Borel map with $x E y \Leftrightarrow f(x) F f(y)$.

Then there is a Borel set $Z \subseteq f[X]$, such that $Z$ and $f[X]$ meet the same $F$-equivalence classes and such that $E \cong(F \mid Z) \times I_{\mathbb{R}}$. 
Proof. Note that if $C$ is an $E$-equivalence class, then $f[C]$ is countable; thus, for some $y \in f[C], f^{-1}(\{y\}) \subseteq C$ is not in $J_{C}$. So define

$$
\begin{aligned}
R(y, x) & \Leftrightarrow f(x)=y \& f^{-1}(\{y\}) \notin J_{[x]_{E}} \\
& \Leftrightarrow f(x)=y \&\left\{x^{\prime}: f(x)=f\left(x^{\prime}\right)\right\} \notin J_{[x]_{E}}
\end{aligned}
$$

so that $R$ is Borel.

Claim. There is a Borel uniformization $R^{*} \subseteq R$, i.e., a Borel set $R^{*} \subseteq R$ such that $\left\{y: \exists x(y, x) \in R^{*}\right\}=\{y: \exists x(y, x) \in R\}$ and $(y, x) \in R^{*} \&\left(y, x^{\prime}\right) \in$ $R^{*} \Rightarrow x=x^{\prime}$.

Granting this claim it follows immediately that $Z=\{y: \exists x(y, x) \in R\}$ is Borel and the function $r: Z \rightarrow X$ given by $r(z)=x \Leftrightarrow R^{*}(z, x)$ is Borel too. Note that $Z$ and $f[X]$ meet the same $F$-equivalence classes.

If

$$
X^{*}=\{x: \exists y(x, y) \in R\}=\left\{x:\left\{x^{\prime}: f(x)=f\left(x^{\prime}\right)\right\} \notin J_{[x]_{E}}\right\}
$$

then $X^{*}$ is also a Borel subset of $X$ and $f$ is a surjection of $X^{*}$ onto $Z$. Also $\forall x \in X \exists z \in Z(f(x) F z)$, so since every $F$-equivalence class is countable, it follows that $\forall x \in X$ (there are at most countably many $z \in Z$ with $f(x) F z)$; so by a standard uniformization theorem, let $g: X \rightarrow Z$ be Borel with $f(x) F g(x), g(x) \in Z$. Let $h: X \rightarrow Z$ be the Borel surjection defined by $h(x)=f(x)$ if $x \in X^{*}$, and $h(x)=g(x)$ if $x \notin X^{*}$. Then $h(x) F f(x)$, so $x E y \Leftrightarrow h(x) F h(y)$ and, for each $z \in Z, h^{-1}[\{z\}] \notin J_{[x]_{E}}$ if $h(x)=z$. Put $H(z, x) \Leftrightarrow h(x)=z$. To each $z \in Z$ assign the following $\sigma$-ideal $I_{z}$ of subsets of $H_{z}$ :

$$
I_{z}=\left\{A \subseteq H_{z}: A \in J_{[x]_{E}}\right\}
$$

where $h(x)=z$. Then all the conditions of Lemma 1.1 are satisfied. (To verify condition (2) note that if $P \subseteq H$ then

$$
\begin{aligned}
z \in P_{I} & \Leftrightarrow P_{z} \in I_{z} \\
& \Leftrightarrow \exists x\left[h(x)=z \&\left\{x^{\prime}:\left(h(x), x^{\prime}\right) \in P\right\} \in J_{\left.[x]_{E}\right]}\right. \\
& \Leftrightarrow \forall x\left[h(x)=z \Rightarrow\left\{x^{\prime}:\left(h(x), x^{\prime}\right) \in P\right\} \in J_{\left.[x]_{E}\right]}\right.
\end{aligned}
$$

so $P_{I}$ is Borel.) Then there is Borel $\varphi: Z \times \mathbb{R} \rightarrow X$ such that for each $z, \varphi_{z}$ is a bijection of $\mathbb{R}$ into $H_{z}$. But then clearly $\varphi$ is a Borel isomorphism of $(F \mid Z) \times I_{\mathbb{R}}$ with $E$.

So it remains to give the

Proof of the claim. The proof is similar to that of 2.4 in [K], so we only give a sketch. Let $\left\{R^{s}\right\}_{s \in \mathbb{N}<N}$ be a family of Borel sets having properties (i)-(iii) (for $R$ ) as in the beginning of the proof of Lemma 1.1. For each $y$ with $\exists x(y, x) \in R$, i.e., $R_{y} \neq \varnothing$, so that actually $R_{y} \notin J_{[x]_{E}}$, where $f(x)=y$, define $\alpha_{y}: \mathbb{N} \rightarrow \mathbb{N}$ inductively so that $\alpha_{y}(n)$ is the least number $m$ for which $R_{y}^{\left(\alpha_{y} \mid n\right)^{\wedge} m}=\left\{x^{\prime}:\left(y, x^{\prime}\right) \in R^{\left(\alpha_{y} \mid n\right)^{\hat{m}} m}\right\} \notin J_{[x]_{E}}$. Let $x_{y}$ be the unique element of $R_{y}^{\alpha}=\bigcap_{n} R_{y}^{\alpha \mid n}$. Put $R^{*}(y, x) \Leftrightarrow x=x_{y}$.

\section{The MAIN Results}

We have first

Theorem 2.1. Let $G$ be a second countable locally compact group and $(g, x) \mapsto$ g.x a Borel action of $G$ on a standard Borel space $X$. Let $E_{G}$ be the induced 
Borel equivalence relation

$$
x E_{G} y \Leftrightarrow \exists g \in G(g . x=y) .
$$

Then if $C=\left\{x:[x]_{E_{G}}\right.$ is countable $\}$ and $U=X \backslash C$, the sets $C, U$ are Borel invariant and there is a Borel set $Z \subseteq U$, which meets every $E_{G} \mid U$-equivalence class in a countable non- $\varnothing$ set and $E_{G} \mid U \cong\left(E_{G} \mid Z\right) \times I_{\mathbb{R}}$. (Notice that $E_{G} \mid Z$ is a countable Borel equivalence relation.)

Proof. For each $E_{G}$-equivalerce class $D$ define the $\sigma$-ideal $J_{D}$ of a subset of $D$ as

$$
A \in J_{D} \Leftrightarrow\{g \in G: g . x \in A\} \text { is meager in } G
$$

where $x \in D$ (this is easily seen to be independent of the choice of $x$ ). Note (see, e.g., [K]) that if $P \subseteq E_{G}$ is Borel, so is $P_{J}=\left\{x: P_{x} \in J_{[x]_{E}}\right\}$. Note now that

$$
\begin{aligned}
{[x]_{E_{G}} \text { is countable } } & \Leftrightarrow G_{x}=\{g \in G: g . x=x\} \text { has countable index in } G \\
& \Leftrightarrow G_{x} \text { is not meager in } G
\end{aligned}
$$

(the last equivalence following from the Baire Category Theorem). So

$$
x \in C \Leftrightarrow\{x\} \notin J_{[x]_{E_{G}}},
$$

thus $C$ (and therefore $U$ ) is Borel.

By the main Theorem 1.2 of $[\mathrm{K}]$, there is a Borel set $Y \subseteq U$ which meets every $E_{G} \mid U$-equivalence class in a countable non- $\varnothing$ set. Since the set

$$
R(x, z) \Leftrightarrow x \in U \& y \in Y \& x E_{G} y
$$

is Borel and has countable sections, while $\forall x \in U \exists y \in Y R(x, y)$, it follows by a standard uniformization theorem that there is a Borel function $f$ such that $\forall x \in U R(x, f(x))$; thus, in particular, $x E_{G} y \Leftrightarrow f(x) F f(y)$, where $F=E_{G}$ । $Y$. All the conditions of Lemma 1.2 are now satisfied, so there is a Borel set $Z \subseteq Y$ meeting every $E_{G} \mid U$-equivalence class in a non- $\varnothing$ countable set and $E_{G} \mid U \cong\left(E_{G} \mid Z\right) \times I_{\mathbf{R}}$.

If $E, E^{\prime}$ are Borel equivalence relations on disjoint standard Borel spaces $X, X^{\prime}$, we denote by $E \oplus E^{\prime}$ their direct sum (i.e., the union of $E, E^{\prime}$ which is a Borel equivalence relation on $X \cup X^{\prime}$ ). It follows from the preceding that every $E_{G}$, where $G$ is second countable locally compact, can be uniquely written as $E \oplus E^{\prime}$, where $E$ is a countable Borel equivalence relation and $E^{\prime}=F \times \mathbb{I}_{\mathbb{R}}$ for some countable Borel equivalence relation $F$.

Since by a result of Feldman-Moore [FM] every countable Borel equivalence relation $E$ is of the form $E=E_{G}$ for some countable group $G$, and so, in particular, $E=E_{F_{\omega}}$, where $F_{\omega}$ is the free group on a countably infinite set of generators, it follows that every $E_{G}$, with $G$ second countable locally compact, is Borel isomorphic to some $E_{F_{\omega} \times \mathbb{R}}$.

For two Borel equivalence relations $E, E^{\prime}$ on $X, X^{\prime}$ respectively, we write

$$
E \subseteq^{i} E^{\prime} \Leftrightarrow \text { there is a Borel invariant } Y \subseteq X^{\prime} \text { with } E \cong E^{\prime} \mid Y .
$$

Since it has been shown in [V] that, for every second countable locally compact group $G$, there is a universal Borel $G$-action, it follows in particular that there is some $E_{G}^{0}$ such that for every $E_{G}, E_{G} \sqsubseteq^{i} E_{G}^{0}$. Thus, in view of the preceding remarks, $E_{G} \sqsubseteq^{i} E_{F_{\omega} \times \mathbb{R}}^{0}$ for any $E_{G}, G$ second countable locally compact. 
Let us note now some simple facts concerning equivalence relations of the form $F \times I_{\mathbb{R}}, F$ a countable Borel equivalence relation.

Proposition 2.2. Let $F, F^{\prime}$ be countable Borel equivalence relations. If $F \leq F^{\prime}$ then $F \times I_{\mathbb{R}} \sqsubseteq^{i} F^{\prime} \times I_{\mathbb{R}}$. In particular, $F \approx^{*} F^{\prime} \Leftrightarrow F \times I_{\mathbb{R}} \cong F^{\prime} \times I_{\mathbb{R}}$.

If $G, G^{\prime}$ are second countable locally compact groups and $E_{G}, E_{G^{\prime}}$ have uncountable equivalence classes, then $E_{G} \leq E_{G^{\prime}} \Leftrightarrow E_{G} \sqsubseteq^{i} E_{G^{\prime}}$ and thus $E_{G} \approx^{*}$ $E_{G^{\prime}} \Leftrightarrow E_{G} \cong E_{G^{\prime}}$.

Proof. Let $X, X^{\prime}$ be the underlying spaces of $F, F^{\prime}$, and let $f: X \rightarrow X^{\prime}$ be Borel such that $x F y \Leftrightarrow f(x) F^{\prime} f(y)$. Since $f$ is countable-to-1, $f[X]=Z$ is Borel and so is $W=[Z]_{F^{\prime}}$. Also clearly, $F \approx^{*} F^{\prime} \mid W$. Then $F \times I_{\mathbb{N}} \cong\left(F^{\prime} \mid W\right) \times$ $I_{\mathbb{N}}$, so $F \times I_{\mathbb{R}} \cong F \times I_{\mathbb{N}} \times I_{\mathbb{R}} \cong\left(F^{\prime} \mid W\right) \times i_{\mathbb{N}} \times I_{\mathbb{R}} \cong\left(F^{\prime} \mid W\right) \times I_{\mathbb{R}}=\left(F^{\prime} \times I_{\mathbb{R}}\right) \mid(W \times \mathbb{R})$. But $W \times \mathbb{R}$ is invariant Borel in $F^{\prime} \times I_{\mathbb{R}}$, so $F \times I_{\mathbb{R}} \sqsubseteq^{i} F^{\prime} \times I_{\mathbb{R}}$. The second assertion follows from the usual Schroeder-Bernstein argument.

If now $E_{G}, E_{G^{\prime}}$ are as in the statement of the proposition, then by Theorem $2.1 E_{G} \cong F \times I_{\mathbb{R}}, E_{G^{\prime}} \cong F^{\prime} \times I_{\mathbb{R}}$, for some countable Borel $F, F^{\prime}$. As $F \leq$ $F \times I_{\mathbb{R}}$ and $F^{\prime} \times I_{\mathbb{R}} \leq F^{\prime}$, we have that $E_{G} \leq E_{G^{\prime}}$ implies $F \leq F^{\prime}$, and so $E_{G} \cong F \times I_{\mathbb{R}} \sqsubseteq^{i} F^{\prime} \times I_{\mathbb{R}} \cong E_{G^{\prime}}$.

Corollary 2.3. The map $F \mapsto F \times I_{\mathbb{R}}$ gives a 1-1 correspondence between countable Borel equivalence relations up to stable isomorphism (or equivalently, bireducibility) and Borel equivalence relations $E_{G}, G$ a second countable locally compact group, with uncountab!e equivalence classes, up to isomorphism (or equivalently, bireducibility).

Finally, we consider $\mathbb{R}$-actions, i.e., flows. It follows from a result of Wagh [W] (see also $[\mathrm{K}]$ ) that, for every Borel action of $\mathbb{R}$ on a standard Borel space $X$, there is $Y \subseteq X$ such that $E_{\mathbb{R}} \mid Y$ is hyperfinite, where a Borel equivalence relation $E$ is hyperfinite if it can be written as $E=\bigcup_{n} E_{n}$, with $E_{0} \subseteq E_{1} \subseteq \ldots$ Borel equivalence relations with finite equivalence classes (thus $E$ is countable).

It is shown in [DJK] that any two hyperfinite nonsmooth Borel equivalence relations are stably isomorphic. It thus follows that we have the following classification.

Theorem 2.4. Let $E_{\mathbb{R}}^{0}, E_{\mathbb{R}}^{1}$ be two Borel equivalence relations induced by Borel $\mathbb{R}$-actions. Let

$$
c_{i}=\operatorname{card}\left(\left\{[x]_{E_{\mathbf{R}}^{i}}:[x]_{E_{\mathbf{R}}^{i}} \text { is a singleton }\right\}\right) .
$$

If $E_{\mathbb{R}}^{0}, E_{\mathbb{R}}^{1}$ are not smooth, then $E_{\mathbb{R}}^{0} \cong E_{\mathbb{R}}^{1} \Leftrightarrow c_{0}=c_{1}$. In particular, all nonsmooth $E_{\mathbb{R}}$ with uncountable equivalence classes are Borel isomorphic.

Proof. Let $X^{0}, X^{1}$ be the spaces of $E_{\mathbb{R}}^{0}, E_{\mathbb{R}}^{1}$. Put

$$
\tilde{X}^{i}=\left\{x \in X^{i}:[x]_{E_{\mathrm{R}}^{i}} \text { is a singleton }\right\} \text {. }
$$

Then $\tilde{X}^{i}$ are Borel, and if $c_{0}=c_{1}, E_{\mathbb{R}}^{0}\left|\tilde{X}^{0}=E_{\mathbb{R}}^{1}\right| \tilde{X}^{1}$. Let $Y^{i}=X^{i} \backslash \tilde{X}^{i}$. As $E_{\mathrm{R}}^{i} \mid \tilde{X}^{i}$ is smooth, $E_{\mathbb{R}}^{i} \mid Y^{i}$ is not smooth. Moreover, each $E_{\mathbb{R}}^{i}$-equivalence class in $Y^{i}$ is uncountable, since the cardinality of $[x]_{E_{\mathrm{R}}^{i}}$ is the same as the index of the stabilizer of $x$ in the action inducing $E_{\mathbb{R}}^{i}$, which is a closed subgroup of $\mathbb{R}$ (see [V]). So $E_{\mathbb{R}}^{i} \mid Y^{i} \cong F_{i} \times I_{\mathbb{R}}$ with $F_{i}=E_{\mathbb{R}}^{i} \mid Z_{i}$ hyperfinite and nonsmooth. So $F_{0} \cong{ }^{s} F_{1} ;$ thus, $E_{\mathbb{R}}^{0}\left|Y^{0} \cong E_{\mathbb{R}}^{0}\right| Y^{1}$. 


\section{REFERENCES}

[B] J. Burgess, A selection theorem for group actions, Pacific J. Math 80 (1979), 333-336.

[DJK] R. Dougherty, S. Jackson, and A. S. Kechris, The structure of hyperfinite Borel equivalence relation, Trans. Amer. Math. Soc. (to appear).

[FHM] J. Feldman, P. Hahn, and C. C. Moore, Orbit structure and countable sections for actions of continuous groups, Adv. in Math. 26 (1979), 186-230.

[FM] J. Feldman and C. C. Moore, Ergodic equivalence relations, cohomology and von Neumann algebras. I, Trans. Amer. Math. Soc. 234 (1977), 289-324.

[K] A. S. Kechris, Countable sections for locally compact group actions, Ergodic Theory Dynamical Systems 12 (1992), 283-295.

[V] V. S. Varadarajan, Groups of automorphisms of Borel spaces, Trans. Amer. Math. Soc. 109 (1963), 191-220.

[W] V. M. Wagh, A descriptive version of Ambrose's representation theorem for flows, Proc. Indian Acad. Sci. Math. Sci. 98 (1988), 101-108.

Department of Mathematics, California Institute of Technology, Pasadena, CaliFORNIA 91125

E-mail address: kechris@caltech.bitnet

kechris@romeo-caltech.edu 